\title{
Submicron Size All-Semiconductor Vertical Cavities with High Q
}

\author{
Abdullah Demir ${ }^{1}$, Doğukan Apaydın², Hamza Kurt ${ }^{2}$ \\ 1. Bilkent University, UNAM - Institute of Materials Science and Nanotechnology, 06800 Ankara, Turkey \\ 2. TOBB University of Economics and Technology, Department of Electrical and Electronics Engineering, 06510 Ankara, Turkey
}

The miniaturization of lasers promises on-chip optical communications and data processing speeds that are beyond the capability of electronics and today's high-speed lasers [1]. Lasers with low-power consumption are one of the most important parts in creating a photonics integrated architecture. This requirement was the motivating force behind the development of small laser and nanolasers. Here, we propose a new method that could be utilized to fabricate such a laser. Oxide-VCSELs require strict control of the oxidation process with significantly reduced reliability for small size, and micropillars have degraded $Q$ with fabrication artifacts for submicron diameter pillars [2]. We propose to use a phase-shifting current-blocking (PSCB) layer serving dual function for a nanocavity device (Fig. 1a) providing both optical- and electrical-confinement via lithographically defined and selectivelybiased buried structures. Phase-shifting leads to optical-confinement tuning by layer thickness control and currentblocking provides electrical-confinement. By modifying the dimensions of these layers, the confinement can be tuned by lithographic means [3]. We studied the electromagnetic wave propagation and analyzed the quality factor (Q) of these cavities based on 3D finite difference time domain (FDTD) calculations.

For optical-confinement, our approach utilizes the effective index model by using thin epitaxial layers. The effective index depends only on the lateral changes in the cavity resonance and the cavity with a longer wavelength has a higher effective index $\left(\Delta \lambda / \lambda_{0}=\Delta \mathrm{n} / \mathrm{n}_{0}\right)$ [4]. The schematic and key components of the studied cavity is shown in Fig. 1a. In our simulations, the structure consists of a $\lambda$-thick GaAs cavity spacer sandwiched between AlAs/GaAs quarter-wavelength distributed Bragg reflectors (DBR) with 35 pairs for the bottom and 5-35 pairs for the top mirror. Although the simulations in this study are realized for a cavity with a resonance at $980 \mathrm{~nm}$, the design approach can be applied to different wavelengths and material systems. We performed a Q-factor comparison for the fundamental mode as a function of the diameter for different confinement strengths (see Fig. $1 \mathrm{~b}$ for 15 pairs of top DBR). The maximum value of $\mathrm{Q}$ is less than 5000 for the studied region. For large confinement $(\Delta \mathrm{n}=0.087$ and 0.053$)$, the resonance wavelength of the fundamental mode (Fig. 1c) shifts to shorter wavelengths and Q-factor is also degraded, which is similar to the case in micropillars [2]. For lower confinement $(\Delta \mathrm{n}=0.022$ and 0.011$)$, there is an overall improvement of $\mathrm{Q}$ and the cavity supports modes with $\mathrm{Q} \sim 4000$ even for submicron diameters. To gain more insight about the Q-factor, we performed a side-by-side comparison of our approach and micropillar cavity. For a submicron diameter $(\mathrm{D}=0.9 \mu \mathrm{m})$, the saturation of Q-factor for the micropillar is reached at $\sim 10^{4}$. For the lithographic method, Q-factor increase to larger than $7 \times 10^{4}$ without saturation, which illustrates the improved photon confinement mechanism of the proposed method compared with the pillar design.
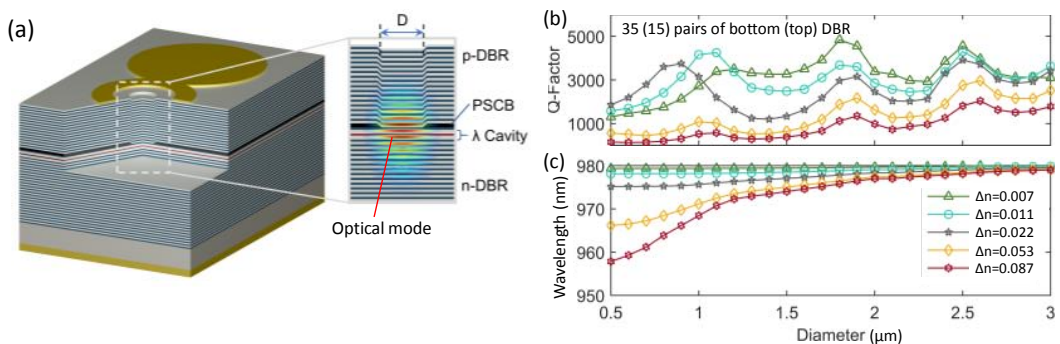

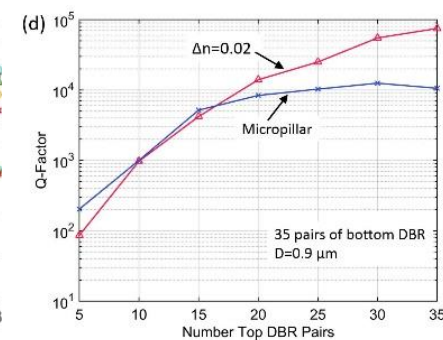

Fig. 1 (a) Lithographic all-semiconductor cavity geometry used in the study. (b) Cavity Q and (c) resonance wavelength of the fundamental mode as a function of the diameter. (d) Q-factor as a function of the number of top DBR pairs for lithographic $(\Delta \mathrm{n}=0.022)$ vs. micropillar cavity for $\mathrm{D}=0.9 \mu \mathrm{m}$.

We have proposed a novel method to enable photon confinement with high Q for submicron diameters. It is a promising approach to create a nanolaser. It can also be utilized to obtain large size, and hence high-power, single transverse mode light sources. This concept can also be extended to arrays of cavities for the implementation of novel nanophotonic devices.

\section{References}

[1] M.T. Hill and M.C. Gather, "Advances in small lasers," Nature Photon. 8, 908 (2014).

[2] G. Lecamp, J. P. Hugonin, and P. Lalanne, "Submicron-diameter semiconductor pillar microcavities with very high quality factors," Appl. Phys. Lett. 90, 091120 (2007).

[3] A. Demir, G. Zhao, and D. Deppe, "Lithographic lasers with low thermal resistance,” Electron. Lett. 46, 1147 (2010).

[4] G. R. Hadley, "Effective index model for vertical-cavity surface-emitting lasers,” Opt. Lett. 20, 1483 (1995). 